\title{
Gout, not hyperuricemia alone, impairs left ventricular diastolic function
}

Jing-Chi Lin ${ }^{1,4}$, Chun-Liang Lin ${ }^{3,4}$, Mien-Cheng Chen ${ }^{5}$, Pey-Jium Chang ${ }^{4}$, Shih-Tai Chang ${ }^{2}$, Chang-Min Chung ${ }^{2}$ and Kuo-Li Pan ${ }^{2,4^{*}}$

\begin{abstract}
Introduction: Gout is a common metabolic disorder characterized by hyperuricemia and chronic inflammation. Previous studies show that hyperuricemia accelerates the occurrence and worsening of cardiovascular disease due to LV remodeling. However, it is still unclear whether hyperuricemia is the sole contributor to organic heart remodeling in patients with gout. In addition, there is a paucity of data regarding the association between LV diastolic function and gout. The objective of this study was to investigate the effects of gout on LV diastolic function.
\end{abstract}

Methods: A total of 173 patients were divided into tertiles based on the following serum uric acid (UA) levels: 1) serum $\cup A \leq 6.5 \mathrm{mg} / \mathrm{dL}(\mathrm{n}=54)$, 2) serum $\cup A>6.5$ to $\leq 8.5 \mathrm{mg} / \mathrm{dL}(\mathrm{n}=59)$, and 3$)$ serum $\cup A>8.5 \mathrm{mg} / \mathrm{dL}$ $(n=60)$.Patients underwent a comprehensive Doppler-echocardiography examination to evaluate LV volume, systolic and diastolic function, and left atrial (LA) volume.

Results: LV diastolic parameters, including diastolic peak early transmitral flow velocity (E), late transmitral flow velocity (A), E/A, peak early diastolic mitral annular velocity (Em), late diastolic annular velocity (Am), Em/Am, E/Em, maximal LA volume index (LAVi) and prevalence of moderate to severe LV diastolic dysfunction were not significantly different between the three groups. Among the population being studied, 108 individuals received a gout diagnosis. Gout patients had greater LV end-systolic dimensions ( $27.08 \pm 4.38 \mathrm{~mm}, \mathrm{p}=0.006)$, higher LV mass index $\left(107.18 \pm 29.51 \mathrm{~g} / \mathrm{m}^{2}, p<0.001\right)$, higher $\mathrm{E} / \mathrm{Em}(10.07 \pm 2.91, \mathrm{p}=0.008)$, and larger maximal LAVi $(16.96 \pm$ $\left.7.39 \mathrm{~mL} / \mathrm{m}^{2}, \mathrm{p}<0.001\right)$ than patients without gout. The prevalence of moderate to severe LV diastolic dysfunction was higher in patients with gout ( $23 \%, p=0.02)$.

Conclusions: Gout, not hyperuricemia alone, is associated with LV diastolic dysfunction and LA volume enlargement.

Keywords: Gout, Hyperuricemia, Diastolic dysfunction, Left atrial volume

\section{Introduction}

Gout is a common metabolic disorder characterized by hyperuricemia and chronic inflammation [1]. Numerous studies have reported elevated uric acid (UA) as a risk factor for coronary heart disease [2, 3], atrial fibrillation [4] and cardiac mortality $[5,6]$. In addition, hyperuricemia is a demonstrated predictor of poor prognosis in various diseases, including acute stroke, congestive heart failure, acute ST-elevation myocardial infarction and chronic renal disease [7-10]. Elevated UA is also associated with

\footnotetext{
* Correspondence: pankuoli64@gmail.com

2Division of Cardiology, Chang Gung Memorial Hospital, Chiayi, Taiwan ${ }^{4}$ Graduate Institute of Clinical Medical Sciences, Chang Gung University, Taoyuan, Taiwan

Full list of author information is available at the end of the article
}

left ventricular (LV) hypertrophy in patients without underlying cardiovascular disease [11] and with diastolic dysfunction in patients with heart failure [12]. Hyperuricemia accelerates the occurrence and worsening of cardiovascular disease due to LV remodeling.

In previous studies it has been observed that cardiovascular events are positively associated with gout. Early evidence reported in the Framingham study found a $60 \%$ increased risk of coronary artery disease (CAD) among gout patients [13]. A Taiwanese study demonstrated that the frequency of gout attacks was associated with an odds ratio of 1.18 for myocardial infarction [14]. The MRFIT study found that gout was associated with a $26 \%$ increased risk of acute myocardial infarction [15]. 
Clinically, the severity of gout, rather than hyperuricemia, is more representative of the chronic inflammation observed in gout patients. Epidemiologically, the study of Kuo et al. demonstrated a link between gout, not hyperuricemia, and a higher risk of death from all causes and cardiovascular diseases [6].

It is still unclear whether hyperuricemia is the sole contributor to organic heart remodeling in patients with gout. Furthermore, there is a paucity of data on the association between LV diastolic function and gout. Therefore, the aim of the current study was to investigate the effects of gout on LV diastolic function.

\section{Methods}

\section{Subjects and study design}

In this prospective study, patients without hyperuricemia, with hyperuricemia, or with gouty arthritis, who underwent echocardiographic examinations, were enrolled between October 2010 and February 2013. Initially, a total of 200 patients were enrolled in this study. Exclusion criteria were the presence of moderate or severe valvular disease, dilated or hypertrophic cardiomyopathy, LV systolic dysfunction (defined as LV ejection fraction (LVEF) $<50 \%$ ), left bundle branch block, second-degree or third-degree atrioventricular conduction block, permanent cardiac pacemaker implantation, atrial arrhythmias (such as atrial fibrillation, atrial flutter, or atrial tachycardia documented by electrocardiography), congenital heart disease, and a history of previous cardiac surgery. Patients were also excluded if they had poor-qualitied echocardiographic images that precluded analysis.

This study was approved by Chang Gung Medical Foundation Institutional Review Board (IRB 100-3022B) and informed consent was obtained from all patients. The clinical data recorded from the enrolled patients included age, sex, height, weight, systolic blood pressure, serum creatinine levels, serum UA levels, and absence or presence of hypertension, diabetes mellitus, hypercholesterolemia, and smoking. Asymptomatic hyperuricemia was defined as UA $\geq 7 \mathrm{mg} / \mathrm{dL}$. The diagnosis of gouty arthritis was made according to the American College of Rheumatology (ACR)/Wallace criteria [16]. Patients who had been diagnosed with gouty arthritis using ACR criteria and received medication were also included in the gouty arthritis group. Hypertension was defined as systolic blood pressure $\geq 140 \mathrm{mmHg}$ and/or diastolic blood pressure $\geq 90 \mathrm{mmHg}$. Additionally, patients who were receiving antihypertensive medication were also considered hypertensive. Diabetes mellitus was defined as a fasting plasma glucose level $\geq 126 \mathrm{mg} / \mathrm{dL}$. Patients receiving oral anti-glucose drugs or insulin for diabetes mellitus control were also considered to have diabetes mellitus. Hypercholesterolemia was defined as either the use of cholesterol-lowering medication or having a total serum cholesterol $\geq 250 \mathrm{mg} / \mathrm{dL}$ in the absence of cholesterol-lowering medication.

\section{Echocardiography}

All subjects received transthoracic echocardiographic examinations at rest in the left lateral decubitus position using a Philips iE33 ultrasound system and S5-1 broadband phased array transducer. The LA diameter in the end-systolic phase, thickness of the interventricular septum (IVS) and posterior wall (PW) in end-diastolic phase, and LV end-diastolic and end-systolic dimensions were all determined using the M-mode in the parasternal long-axis view according to the recommendations of the American Society of Echocardiography [6]. The left ventricular ejection fraction (LVEF), which means LV systolic function, was calculated using Simpson's method [6]. The LV mass was estimated by the Devereux formula [17]. Then, the LV mass index was obtained as the LV mass/body surface area (BSA). Mitral inflow velocities were evaluated with a 1- to 2-mm sample volume placed at the mitral valve tip by pulse-wave tissue Doppler imaging (TDI) in the apical four-chamber view. Diastolic peak early (E) and late (A) transmitral flow velocity and the $\mathrm{E}$ to $\mathrm{A}$ ratio $(\mathrm{E} / \mathrm{A})$ were measured. In the apical four-chamber view, the peak early diastolic mitral annular velocity $(\mathrm{Em})$ and peak late diastolic annular velocity (Am) were obtained by pulse-wave TDI at the septal site of the mitral annulus. The E/Em ratio was used as an index of LV filling pressure [18]. LV enddiastolic volume (LVEDV) and LV end-systolic volume (LVESV) were measured in the apical four-chamber and two-chamber views using the biplane modified Simpson's method. Then, the LVEDV and LVESD indexes were calculated as LVEDV/BSA and LVESV/BSA. Maximal left atrial volume (LAV) was also measured in the apical four-chamber and two-chamber views using the biplane modified Simpson's method [19-21]. Maximal LAV was measured just before mitral valve opening, and the maximal LAVi was calculated as maximal LAV/BSA. All echocardiographic parameters were measured from an average of three beats. E/A and E/Em are the parameters of LV diastolic dysfunction clinically. According to the value of E/A and E/Em, moderate to severe LV diastolic function was defined as (1) a pseudonormal pattern: $0.75 \leq \mathrm{E} / \mathrm{A}$ ratio $<1.5$ and $\mathrm{E} / \mathrm{Em}$ ratio $>10$ and (2) a restrictive pattern: $\mathrm{E} / \mathrm{A}$ ratio $\geq 1.5$ and $\mathrm{E} / \mathrm{Em}$ ratio $>10$ [22].

\section{Statistical analysis}

Statistical analysis was performed using SPSS 18.0 statistical software (SPSS Inc., Chicago, IL, USA). Continuous data are presented as mean \pm standard deviation (SD), and dichotomous data as number and percentage. Comparisons of continuous variables between groups were performed using analysis of variance (ANOVA) or the 
unpaired two-tailed $t$ test. Categorical variables were compared using the chi square $\left(x^{2}\right)$ test. Multiple regression analyses were performed to determine the associations between gout and LV diastolic functional parameters, including E/A, Em, Am, E/Em, and maximal LAV. The multivariate model was adjusted for age, sex, UA, creatinine, diabetes mellitus, hypertension, hypercholesterolemia, and smoking. Statistical significance was set at $p<0.05$. In 20 randomly selected subjects, the interobserver and intraobserver variability in E/Em and maximal LAV measurements were assessed by the coefficient of variation, where differences between measurements were expressed as the ratio of the SD to the mean. Interobserver variability was assessed by two independent observers and intraobserver variability by one observer twice within a two-week period. The interobserver variability of $\mathrm{E} / \mathrm{Em}$ was $3.3 \%$ and that of maximal LAV was $2.2 \%$. The intraobserver variability of E/ Em was $1.8 \%$ and that of maximal LAV was $1.5 \%$.

\section{Results}

Baseline characteristics of patients by UA tertile

A total of 27 patients were excluded because of the presence of LV systolic dysfunction $(n=2)$, hypertrophic cardiomyopathy $(\mathrm{n}=2)$, permanent cardiac pacemaker implantation $(\mathrm{n}=2)$, atrial fibrillation $(\mathrm{n}=2)$, and poor echocardiographic images that precluded analysis $(n=19)$. Thus, the final study population consisted of 173 patients, who were divided into tertiles by serum level of UA as follows: group 1: serum UA $\leq 6.5 \mathrm{mg} / \mathrm{dL}(\mathrm{n}=54)$; group 2 : serum UA $>6.5$ to $\leq 8.5 \mathrm{mg} / \mathrm{dL} \quad(\mathrm{n}=59)$; and group 3 : serum UA $>8.5 \mathrm{mg} / \mathrm{dL}(\mathrm{n}=60)$. The mean UA levels of these three groups were $5.03 \pm 1.07,7.63 \pm 0.52$, and $10.05 \pm 1.17 \mathrm{mg} / \mathrm{dL}$, respectively $(p<0.001)$. The clinical characteristics of the study population are listed in Table 1. There were no significant differences between the three groups in age, gender or creatinine levels, history of diabetes mellitus, hypertension or hypercholesterolemia, or cigarette smoking. Among the echocardiographic parameters, there were no significantly different LV volume parameters between the three groups, including LV enddiastolic dimensions, LV end-systolic dimensions, LVEDV, LVEDV index, LVESV, LVESV index, and LV systolic function. However, patients grouped by tertiles of increasing UA were significantly associated with a graded increase in $\mathrm{LV}$ wall thickness, LV mass, and LV mass index. LV diastolic parameters including E, A, E/A, Em, Am, Em/ $\mathrm{Am}, \mathrm{E} / \mathrm{Em}$, maximal LAVi, and prevalence of moderate to severe LV diastolic dysfunction were not significantly different between the three groups.

\section{LV organic remodeling and diastolic dysfunction in patients with gout}

As there were no obvious findings in relation to uricacid-based grouping, we studied our patients according to diagnosis of gouty arthritis, asymptomatic hyperuricemia and normo-uricemia. Among the study population, 108 individuals received a diagnosis of gout: 65 patients $(60 \%)$ received uricostatic agents, $15(14 \%)$ received uricosuric agents and $77(71 \%)$ received colchicines. Table 1 shows the baseline characteristics of individuals with and without gout. There were no significant differences in age or gender, history of diabetes mellitus, hypertension or hypercholesterolemia, or cigarette smoking between the patients with and without gout. The patients with gout had significantly higher serum UA levels than those without gout $(8.19 \pm 2.04 \mathrm{mg} / \mathrm{dL}, p<0.001)$. Furthermore, patients with gout had significantly worse renal function than those without gout (creatinine $1.34 \pm 1.09 \mathrm{mg} / \mathrm{dL}$, $p<0.001$ and estimated glomerular filtration rate (eGFR) $75.05 \pm 34.2 \mathrm{ml} / \mathrm{min}, p=0.059$ ).

The echocardiographic parameters of patients with and without gout are summarized in Table 1 . Patients with gout had a significantly thicker IVS $(10.49 \pm 1.66 \mathrm{~mm}, p=$ $0.004)$ and PW than those without gout $(10.93 \pm 1.64 \mathrm{~mm}$, $p=0.006)$. The LV mass was significantly larger in patients with gout than in those without $(193.90 \pm 52.59$ vs. $\left.164.54 \pm 47.97 \mathrm{~g} / \mathrm{m}^{2}, p<0.001\right)$. The LV mass index in the patients with gout was also significantly higher than in those without gout $\left(107.18 \pm 29.51\right.$ vs. $95.4 \pm 26.57 \mathrm{~g} / \mathrm{m}^{2}$, $p=0.009$ ). Additionally, patients with gout had greater LV end-diastolic dimensions (LVEDd) than patients without gout $(48.27 \pm 5.53$ vs $46.36 \pm 5.52 \mathrm{~mm}, p=0.031)$. There were no significant differences in LV end-systolic dimensions (LVESd), LVEDV index, and LVESV index between the patients with and without gout. Additionally, there were no significant differences in LV systolic function between patients with and without gout. Among the LV diastolic functional parameters, the $\mathrm{E}, \mathrm{A}$, and $\mathrm{E} / \mathrm{A}$ ratio were not significantly different between patients with and without gout. The patients with gout had significantly lower Em than those without gout $(7.03 \pm 2.02$ vs. $8.45 \pm 2.7$, $p=0.001)$. The Am was not significantly different between groups. The $\mathrm{Em} / \mathrm{Am}$ ratio was lower in patients with gout $(0.82 \pm 0.31, p<0.001)$, and the $\mathrm{E} / \mathrm{Em}$ ratio was higher in the patients with gout $(10.07 \pm 2.91, p=$ 0.008). Furthermore, the maximal LAVi of the patients with gout was significantly larger than that of the patients without gout $\left(16.96 \pm 7.39 \mathrm{~mL} / \mathrm{m}^{2}, p<0.001\right)$. Finally, the prevalence of moderate to severe LV diastolic dysfunction was higher in patients with gout than in patients without gout ( $23 \%$ vs. $8 \%, p=0.016$ ). There were significant differences between the normouricemia and hyperuricemia group, in uric acid, creatinine $(0.83 \pm 0.19$ vs. $1.01 \pm 0.13 \mathrm{mg} / \mathrm{dL}, p<0.001)$, IVS $(9.37 \pm$ 1.61 vs. $10.27 \pm 1.85 \mathrm{~mm}, p=0.41)$ and LV mass (154.11 \pm 48.92 vs. $177.80 \pm 41.77 \mathrm{~g} / \mathrm{m}, p=0.42$ ). There were no further statistically significant differences between the normouricemia and hyperuricemia groups (data not shown). 
Table 1 Baseline characteristics of patients grouped by tertiles of serum uric acid and gout

\begin{tabular}{|c|c|c|c|c|c|c|c|c|c|c|}
\hline Variables & $\begin{array}{l}\text { First }(<6.5 \mathrm{mg} / \mathrm{dL}) \\
(\mathrm{n}=54)\end{array}$ & $\begin{array}{l}\text { Second }(>6.5 \text { and } \\
<8.5 \mathrm{mg} / \mathrm{dL})(\mathrm{n}=59)\end{array}$ & $\begin{array}{l}\text { Third }(>8.5 \mathrm{mg} / \mathrm{dL}) \\
(\mathrm{n}=60)\end{array}$ & $P$ value & $\begin{array}{l}\text { Normo-uricemia } \\
(n=35)\end{array}$ & $\begin{array}{l}\text { Hyperuricemia } \\
(n=30)\end{array}$ & Gout $(n=108)$ & $P$ value & $\begin{array}{l}\text { Non-gout } \\
(n=65)\end{array}$ & $P$ value \\
\hline Age (years) & $56.86 \pm 15.36$ & $55.79 \pm 11.68$ & $58.44 \pm 13.38$ & 0.56 & $53.54 \pm 15.64$ & $56.96 \pm 16.07$ & $58.20 \pm 11.76$ & 0.20 & $55.09 \pm 16.17$ & 0.154 \\
\hline Men & $42(78 \%)$ & $54(92 \%)$ & $54(90 \%)$ & 0.064 & $26(74 \%)$ & $27(90 \%)$ & $97(90 \%)$ & 0.06 & $50(81 \%)$ & 0.079 \\
\hline Body surface area $\left(\mathrm{m}^{2}\right)$ & $1.70 \pm 0.20$ & $1.83 \pm 0.22^{*}$ & $1.81 \pm 0.18^{*}$ & 0.002 & $1.68 \pm 0.19$ & $1.77 \pm 0.16$ & $1.82 \pm 0.20^{*}$ & 0.003 & $1.72 \pm 0.18^{*}$ & 0.004 \\
\hline Uric acid (mg/dL) & $5.03 \pm 1.07$ & $7.63 \pm 0.52^{*}$ & $10.05 \pm 1.17^{* \dagger}$ & $<0.001$ & $4.96 \pm 1.04$ & $8.87 \pm 1.42^{*}$ & $8.19 \pm 2.04^{*}$ & $<0.001$ & $6.68 \pm 2.3^{* *}$ & $<0.001$ \\
\hline Creatinine (mg/dL) & $1.0 \pm 0.43$ & $1.19 \pm 0.38$ & $1.34 \pm 1.4$ & 0.115 & $0.83 \pm 0.19$ & $1.01 \pm 0.13$ & $1.34 \pm 1.09^{*}$ & 0.006 & $0.9 \pm 0.19^{* *}$ & $<0.001$ \\
\hline eGFR & $81.68 \pm 32.80$ & $81.33 \pm 34.91$ & $74.53 \pm 31.12$ & 0.41 & $90.14 \pm 30.08$ & $80.67 \pm 29.21$ & $75.05 \pm 34.20$ & 0.059 & $85.77 \pm 29.83^{* *}$ & 0.038 \\
\hline Diabetes mellitus & $3(6 \%)$ & $2(3 \%)$ & $6(10 \%)$ & 0.322 & $2(6 \%)$ & $3(10 \%)$ & $6(6 \%)$ & 0.60 & $5(8 \%)$ & 0.492 \\
\hline Hypertension & $11(20 \%)$ & 12 (20 \%) & $15(25 \%)$ & 0.781 & $8(23 \%)$ & $8(27 \%)$ & 22 (20 \%) & 0.78 & $16(26 \%)$ & 0.362 \\
\hline Hypercholesterolemia & $14(26 \%)$ & $20(34 \%)$ & 19 (32\%) & 0.641 & $17(49 \%)$ & $13(43 \%)$ & $45(42 \%)$ & 0.79 & 17 (27 \%) & 0.493 \\
\hline Smoking & $6(11 \%)$ & $13(22 \%)$ & $6(10 \%)$ & 0.123 & $5(14 \%)$ & $5(17 \%)$ & $15(14 \%)$ & 0.95 & $10(16 \%)$ & 0.657 \\
\hline \multicolumn{11}{|c|}{ LV dimensions and volume } \\
\hline IVS (mm) & $9.51 \pm 1.32$ & $10.65 \pm 1.8^{*}$ & $10.45 \pm 1.82^{*}$ & 0.001 & $9.37 \pm 1.61$ & $10.27 \pm 1.85$ & $10.49 \pm 1.66^{*}$ & 0.004 & $9.79 \pm 1.81^{* *}$ & 0.017 \\
\hline LVEDd (mm) & $46.76 \pm 5.94$ & $48.12 \pm 4.97$ & $47.79 \pm 5.72$ & 0.404 & $45.80 \pm 6.11$ & $47.15 \pm 4.47$ & $48.27 \pm 5.53$ & 0.06 & $46.36 \pm 5.52^{* *}$ & 0.031 \\
\hline PW (mm) & $9.85 \pm 1.35$ & $11.15 \pm 1.52^{*}$ & $10.94 \pm 1.86^{*}$ & $<0.001$ & $9.89 \pm 1.56$ & $10.64 \pm 1.71$ & $10.93 \pm 1.64^{*}$ & 0.006 & $10.21 \pm 1.66^{* *}$ & 0.007 \\
\hline LVESd (mm) & $26.37 \pm 4.55$ & $27.05 \pm 4.29$ & $27.04 \pm 4.36$ & 0.643 & $25.64 \pm 4.77$ & $27.31 \pm 3.77$ & $27.08 \pm 4.38$ & '0.19 & $26.32 \pm 4.43$ & 0.255 \\
\hline LV mass $(\mathrm{g} / \mathrm{m})$ & $159.6 \pm 44.48$ & $195.18 \pm 48.37^{*}$ & $192.24 \pm 56.28^{*}$ & $<0.001$ & $154.11 \pm 48.92$ & $177.80 \pm 41.77$ & $193.90 \pm 52.59$ & ${ }^{*}<0.001$ & $164.54 \pm 47.97^{* *}$ & $<0.001$ \\
\hline LV mass index $\left(\mathrm{g} / \mathrm{m}^{2}\right)$ & $93.78 \pm 25.09$ & $107.57 \pm 28.39^{*}$ & $106.2 \pm 30.61^{*}$ & 0.019 & $91.14 \pm 27.03$ & $100.56 \pm 24.09$ & $107.18 \pm 29.51$ & ${ }^{*} 0.01$ & $95.4 \pm 26.57^{* *}$ & 0.009 \\
\hline LVEDV (mL) & $69.33 \pm 19.61$ & $77.29 \pm 20.37$ & $72.75 \pm 20.21$ & 0.11 & $66.97 \pm 20.19$ & $68.80 \pm 19.80$ & $76.49 \pm 19.81^{*}$ & 0.02 & $67.73 \pm 20.36^{* *}$ & 0.008 \\
\hline LVEDV index $\left(\mathrm{mL} / \mathrm{m}^{2}\right)$ & $40.83 \pm 11.46$ & $42.29 \pm 10.81$ & $40.11 \pm 10.29$ & 0.538 & $39.55 \pm 10.33$ & $38.51 \pm 9.87$ & $42.28 \pm 11.12$ & 0.15 & $39.09 \pm 10.3$ & 0.066 \\
\hline LVESV (mL) & $19.59 \pm 8.14$ & $22.12 \pm 8.55$ & $20.07 \pm 7.48$ & 0.205 & $18.54 \pm 7.28$ & $19.67 \pm 9.25$ & $21.56 \pm 7.91$ & 0.12 & $19.26 \pm 8.35$ & 0.105 \\
\hline LVESV index $\left(\mathrm{mL} / \mathrm{m}^{2}\right)$ & $11.62 \pm 5.19$ & $12.1 \pm 4.76$ & $11.07 \pm 4.03$ & 0.404 & $10.96 \pm 3.91$ & $10.93 \pm 4.84$ & $11.98 \pm 4.82$ & 0.37 & $11.08 \pm 4.4$ & 0.265 \\
\hline \multicolumn{11}{|l|}{ LV systolic function } \\
\hline LVEF (\%) & $73.99 \pm 9.2$ & $74.28 \pm 7.22$ & $73.82 \pm 7.26$ & 0.95 & $72.57 \pm 5.42$ & $72.50 \pm 6.98$ & $71.95 \pm 6.08$ & 0.83 & $73.67 \pm 8.84$ & 0.671 \\
\hline \multicolumn{11}{|l|}{ LV diastolic function } \\
\hline$E(\mathrm{~m} / \mathrm{s})$ & $0.71 \pm 0.15$ & $0.66 \pm 0.13$ & $0.67 \pm 0.17$ & 0.117 & $0.78 \pm 0.15$ & $0.65 \pm 0.14$ & $0.67 \pm 0.15$ & 0.31 & $0.69 \pm 0.15$ & 0.475 \\
\hline$A(m / s)$ & $0.74 \pm 0.2$ & $0.73 \pm 0.2$ & $0.75 \pm 0.19$ & 0.872 & $0.71 \pm 0.17$ & $0.75 \pm 0.22$ & $0.74 \pm 0.19$ & 0.74 & $0.74 \pm 0.2$ & 0.853 \\
\hline E/A & $1.03 \pm 0.35$ & $0.99 \pm 0.36$ & $0.95 \pm 0.34$ & 0.514 & $1.05 \pm 0.38$ & $0.92 \pm 0.30$ & $0.98 \pm 0.34$ & 0.32 & $0.99 \pm 0.36$ & 0.895 \\
\hline $\mathrm{Em}(\mathrm{cm} / \mathrm{s})$ & $8.12 \pm 2.82$ & $7.34 \pm 2.22$ & $7.24 \pm 2.04$ & 0.102 & $8.79 \pm 3.04$ & $7.94 \pm 2.15$ & $7.03 \pm 2.02^{*}$ & $<0.001$ & $8.45 \pm 2.7^{* *}$ & 0.001 \\
\hline $\mathrm{Am}(\mathrm{cm} / \mathrm{s})$ & $8.42 \pm 1.57$ & $8.93 \pm 1.74$ & $9.14 \pm 2.2$ & 0.117 & $8.24 \pm 1.54$ & $9.01 \pm 2.14$ & $8.98 \pm 1.87$ & 0.11 & $8.69 \pm 1.88$ & 0.414 \\
\hline $\mathrm{Em} / \mathrm{Am}$ & $1.02 \pm 0.5$ & $0.86 \pm 0.38$ & $0.85 \pm 0.34$ & 0.05 & $1.14 \pm 0.57$ & $0.94 \pm 0.40$ & $0.82 \pm 0.31^{*}$ & $<0.001$ & $1.05 \pm 0.52^{* *}$ & 0.001 \\
\hline
\end{tabular}


Table 1 Baseline characteristics of patients grouped by tertiles of serum uric acid and gout (Continued)

\begin{tabular}{|c|c|c|c|c|c|c|c|c|c|c|}
\hline E/Em & $9.49 \pm 3.05$ & $9.47 \pm 2.48$ & $9.67 \pm 3.12$ & 0.918 & $8.58 \pm 2.07$ & $8.79 \pm 3.31$ & $10.07 \pm 2.91^{*}$ & 0.008 & $8.72 \pm 2.74^{* *}$ & 0.004 \\
\hline Maximal LAVi $\left(\mathrm{mL} / \mathrm{m}^{2}\right)$ & $12.94 \pm 5.80$ & $16.45 \pm 6.59^{*}$ & $15.77 \pm 7.18$ & 0.013 & $11.49 \pm 3.36$ & $12.71 \pm 4.20$ & $16.96 \pm 7.39 * f$ & $<0.001$ & $20.34 \pm 4.86^{* *}$ & $<0.001$ \\
\hline $\begin{array}{l}\text { Moderate-to-severe LV diastolic } \\
\text { dysfunction }\end{array}$ & $7(13 \%)$ & $9(15 \%)$ & $14(23 \%)$ & 0.301 & $2(6 \%)$ & $3(10 \%)$ & $25(23 \%)$ & 0.02 & $5(8 \%)^{* *}$ & 0.016 \\
\hline
\end{tabular}

Data are expressed as mean \pm standard deviation or number (percentage). ${ }^{*} P<0.05$ versus group with uric acid $(\mathrm{UA}) \leq 6.5 \mathrm{mg} / \mathrm{dL}$ group or normo-uricemic group. ${ }^{* *} P<0.05$ compared with gout group. ${ }^{\dagger} P<0.05$ versus group with $U A>6.5$ and $\leq 8.5 \mathrm{mg} / \mathrm{Dl}$ or the hyperuricemic group. Moderate-to-severe LV diastolic dysfunction: (1) pseudonormal pattern: $0.75 \leq \mathrm{E} / \mathrm{A}$ ratio $<1.5$ and $\mathrm{E} / \mathrm{Em}$ ratio $>10$ and (2) restrictive pattern: $\mathrm{E} / \mathrm{A}$ ratio $\geq 1.5$ and $\mathrm{E} / \mathrm{Em}$ with $U A>6.5$ and $\leq 8.5 \mathrm{mg} / \mathrm{Dl}$ or the hyperuricemic group. Moderate-to-severe $L V$ diastolic dysfunction: (1) pseudonormal pattern: $0.75 \leq \mathrm{E} / \mathrm{A}$ ratio $<1.5$ and $\mathrm{E} / \mathrm{Em}$ ratio $>10$ and $(2)$ restrictive pattern: $\mathrm{E} / \mathrm{A}$ ratio $\geq 1.5$ and $\mathrm{E} / \mathrm{Em}$
ratio $>10$. eGFR estimated glomerular filtration rate, $A$ late diastolic peak transmitral flow velocity, Am peak late diastolic annular velocity, $E$ early diastolic peak transmitral flow velocity, Em peak early diastolic mitral annular velocity, IVS interventricular septum, LAVi left atrial volume index, LV left ventricle, LVEDd LV end-diastolic diameter, LVEDV LV end-diastolic volume, LVEF LV ejection fraction, LVESd LV end-systolic diameter, LVESV LV end-systolic volume, $P W$ posterior wall thickness 
On multivariate analysis, the associations between gout and LV diastolic functional parameters, including $\mathrm{Em}, \mathrm{Am}, \mathrm{Em} / \mathrm{Am}, \mathrm{E} / \mathrm{Em}$ and maximal LAVi, were significant when controlled for age, sex, UA, creatinine, diabetes mellitus, hypertension, hypercholesterolemia and smoking in the study groups (Table 2).

\section{Discussion}

The major finding of this study was that gout impacts LV diastolic dysfunction and LA volume enlargement. LV diastolic remodeling may be a predictor of adverse cardiac events in gout patients. LV diastolic dysfunction refers to an abnormality of diastolic distensibility, filling, or relaxation of the LV [23]. LV diastolic dysfunction has major effects on the LA, which is a transporting chamber that receives blood from the pulmonary veins and conveys it to the LV through both passive and active diastolic filling [24, 25]. Echocardiographic evidence of diastolic dysfunction is an independent risk factor for heart failure [26]. LV diastolic dysfunction increases atrial afterload, atrial stretch, and atrial wall stress as a result of dilation, which can promote atrial fibrillation. In the study of Tsang et al., more severe diastolic dysfunction and increased LAVi were associated with an increased risk of incident atrial fibrillation [27]. In addition, diabetes mellitus patients with moderate to severe diastolic dysfunction have an increased risk of incident atrial fibrillation [28]. The severity of LV diastolic dysfunction and LA enlargement can predict cardiovascular events.

Elevated serum UA levels are associated with diastolic dysfunction in chronic heart failure patients with LVEF

Table 2 Multivariate regression analysis of the association between left ventricular diastolic functional parameters and the gout study groups $(n=173)$

\begin{tabular}{|c|c|c|c|c|}
\hline \multirow[t]{2}{*}{ Variable } & \multicolumn{2}{|c|}{ Unadjusted $^{a}$} & \multicolumn{2}{|c|}{ Model $1^{\mathrm{b}}$} \\
\hline & $\bar{B}$ & $P$ & $\bar{B}$ & $p$ \\
\hline \multicolumn{5}{|l|}{$\overline{E / A}$} \\
\hline Gout & -0.01 & 0.894 & 0.077 & 0.237 \\
\hline \multicolumn{5}{|c|}{$\mathrm{Em}(\mathrm{cm} / \mathrm{s})$} \\
\hline Gout & -0.282 & $<0.001$ & -0.208 & 0.002 \\
\hline \multicolumn{5}{|c|}{$\operatorname{Am}(\mathrm{cm} / \mathrm{s})$} \\
\hline Gout & 0.062 & 0.414 & -0.019 & 0.815 \\
\hline \multicolumn{5}{|l|}{$\mathrm{Em} / \mathrm{Am}$} \\
\hline Gout & -0.257 & 0.001 & -0.163 & 0.016 \\
\hline \multicolumn{5}{|l|}{$\mathrm{E} / \mathrm{Em}$} \\
\hline Gout & 0.214 & 0.005 & 0.172 & 0.02 \\
\hline \multicolumn{5}{|c|}{ Maximal LAVi $\left(\mathrm{mL} / \mathrm{m}^{2}\right)$} \\
\hline Gout & 0.35 & $<0.001$ & 0.294 & $<0.001$ \\
\hline
\end{tabular}

Including gout. ${ }^{\mathrm{b}}$ Adjusted for age, sex, uric acid, creatinine, diabetes mellitus, hypertension, hyperlipidemia, and smoking. A late diastolic peak transmitral flow velocity, $A m$ peak late diastolic annular velocity, $E$ early diastolic peak transmitral flow velocity, Em peak early diastolic mitral annular velocity, LAVi left atrial volume index
$<45 \%$, due to free radical-mediated endothelial damage resulting from increased xanthine oxidase activity [12]. High UA levels were associated with increased LV mass, end-diastolic LV dimensions, and IVS thickness compared with patients with low UA levels in the present study (Table 1) and that of Krishnan et al. [29]. A possible mechanism to explain this observed relationship is the accumulation of reactive oxygen species due to upregulation of xanthine oxidase in hyperuricemia.

Gout is a metabolic disorder characterized by hyperuricemia and a type of chronic inflammatory arthritis induced by deposition of monosodium urate crystals in the synovial fluid and other tissues [1]. Accumulating evidence from previous studies supports the existence of a repetitive and progressive state of inflammatory activation that is strongly associated with the progression of ventricular diastolic dysfunction and characterized by the intense release and activation of circulating cytokines $[30,31]$. Whether hyperuricemia alone contributes to LV organic and functional remodeling in gout patients remains contentious. The current study is the first to evaluate LV diastolic function in gout patients and to determine the role of chronic gout-related inflammation in LV diastolic dysfunction remodeling. In the present study, LV diastolic functional parameters, including E/A, $\mathrm{Em}, \mathrm{Am}, \mathrm{E} / \mathrm{Em}$, and maximal $\mathrm{LAVi}$, were not significantly different between the groups with low and high serum UA. Furthermore, there was no association between serum UA and moderate-to-severe LV diastolic dysfunction (Table 1).

Interestingly, lower Em and higher E/Em were detected in patients with gout than in those without gout, and the prevalence of moderate-to-severe LV diastolic dysfunction was higher in patients with gout than those without gout ( $23 \%$, vs. $8 \%, p=0.016$; Table 1 ); however, there was no difference between those with normo-uricemia and hyperuricemia. $\mathrm{E} / \mathrm{Em}$ is an excellent predictor of LV filling pressure and a parameter of LV diastolic function [32, 33]. A possible explanation for these observations is that patients with gout present with monosodium urate crystalmediated inflammatory cytokines including tumor necrosis factor- $\alpha$ (TNF- $\alpha$ ) and interleukin- $1 \beta$ (IL-1 $\beta$ ), which have been implicated in the pathogenesis of myocardial dysfunction [34]. Maximal LAVi was also larger in patients with gout than those without gout and there was no difference between the normo-uricemia and hyperuricemia group. LA enlargement is primarily the result of pressure or volume overload, and can serve as an index of atrial remodeling [35]. In our study gout was an independent factor of impaired LV diastolic dysfunction, including decreased $\mathrm{Em}$, decreased $\mathrm{Em} / \mathrm{Am}$, and increased $\mathrm{E} / \mathrm{Em}$ (Table 2). Maximal LAVi was negatively associated with Em $(r=-0.225, p=0.018)$ and positively associated with $\mathrm{E} / \mathrm{Em}(r=0.343, p<0.001)$ (Fig. 1). Gout impairs LV 


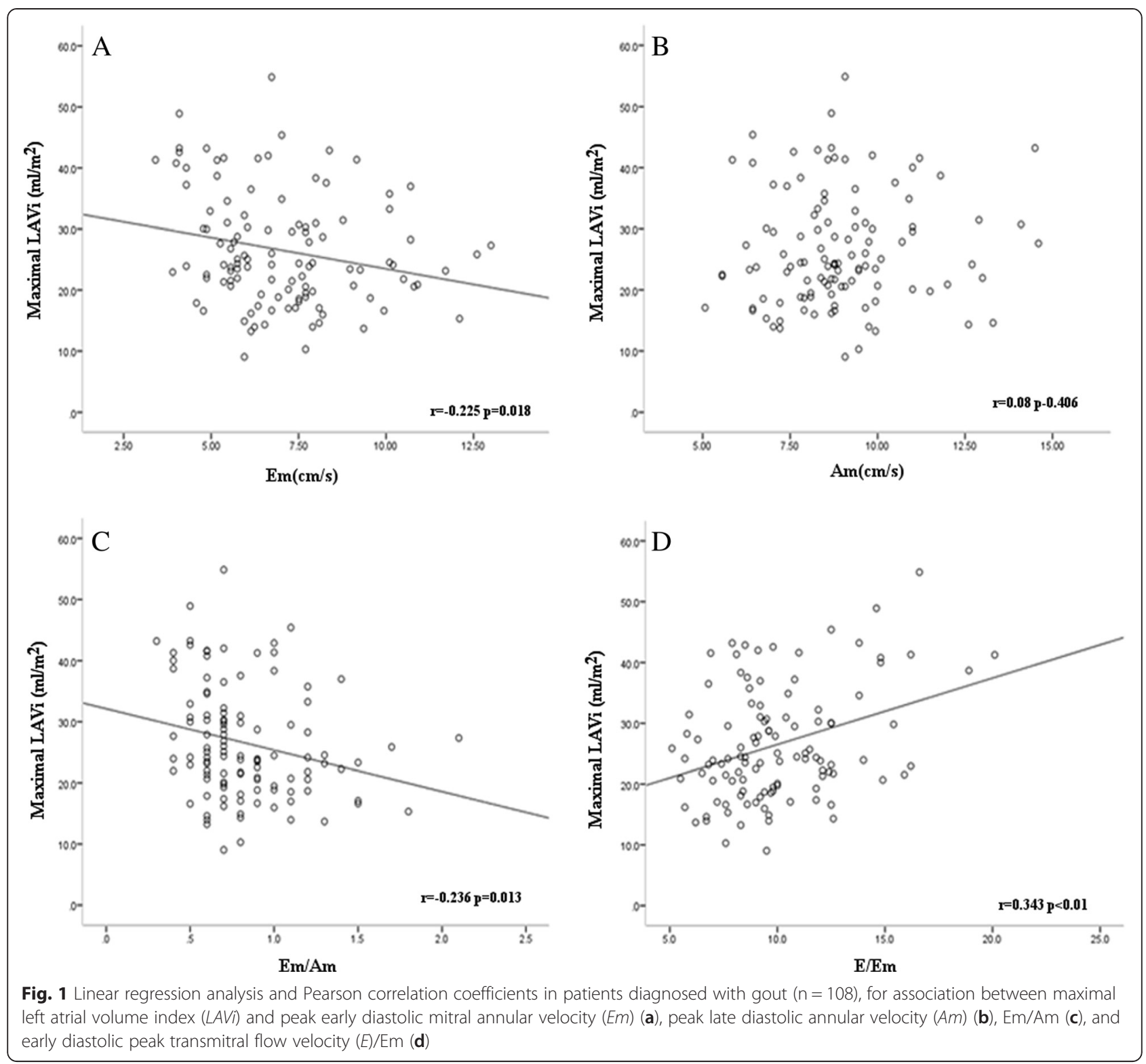

compliance, which increases LV filling pressure. During ventricular diastole, the LA is directly exposed to LV pressure through the open mitral valve. Consequently, LA pressure increases in order to maintain adequate LV filling, which results in increased LA wall tension and ultimately LA enlargement. Therefore, enlarged LA volume may reflect the severity of diastolic dysfunction in gouty arthritis patients. These results suggest that in patients with gout, LV diastolic remodeling might be associated with the inflammation mediated by monosodium urate crystals rather than isolated hyperuricemia.

There are several limitations in the present study. First, further follow-up cohort studies are necessary to demonstrate whether LV diastolic remodeling is predictive of adverse cardiac events in patients with gout.
Second, the biological link between gout and LV diastolic dysfunction remains unclear; our current hypotheses and results require further clarification through additional studies.

\section{Conclusion}

Gout, rather than hyperuricemia, is associated with LV diastolic dysfunction and LA volume enlargement. LV diastolic remodeling may be a predictor of adverse cardiac events in patients with gout.

\section{Abbreviations}

BSA: body surface area; IVS: interventricular septum; LV: left ventricular; LVEF: left ventricular ejection fraction; LVEDV: left ventricular end-diastolic volume; LVESV: left ventricular end-systolic volume; LA: left atrial; LAVi: left atrial volume 
index; LAV: ILeft atrial volume; PW: posterior wall; TDI: tissue Doppler imaging; UA: uric acid.

\section{Competing interests}

The authors declare no conflict of interest or funding support for this study.

\section{Authors' contributions}

JCL and KLP conceived, designed and performed the experiment, analyzed the data and wrote the manuscript. CLL conceived and designed the experiments, and wrote the manuscript. PJC conceived and designed the experiments, and revised the manuscript. STC and CMC performed the experiments and participated in drafting the manuscript. MCC analyzed the data and participated in drafting the manuscript. All authors read and approved the final manuscript.

\section{Acknowledgements}

We would like to thank Chien-Hui Hung and Chung-Sheng Shi, teachers at the Graduate Institute of Clinical Medical Sciences, Chang Gung University, for editing assistance.

\section{Author details}

'Division of Allergy and Immunology and Rheumatology, Chang Gung Memorial Hospital, Chiayi, Taiwan. ${ }^{2}$ Division of Cardiology, Chang Gung Memorial Hospital, Chiayi, Taiwan. ${ }^{3}$ Division of Nephrology, Chang Gung Memorial Hospital, Chiayi, Taiwan. ${ }^{4}$ Graduate Institute of Clinical Medical Sciences, Chang Gung University, Taoyuan, Taiwan. ${ }^{5}$ Division of Cardiology, Kaohsiung Chang Gung Memorial Hospital, Kaohsiung, Taiwan.

\section{Received: 14 July 2015 Accepted: 29 October 2015} Published online: 14 November 2015

\section{References}

1. Neogi T. Gout. N Engl J Med. 2011;364(5):443-52.

2. Kim SY, Guevara JP, Kim KM, Choi HK, Heitjan DF, Albert DA. Hyperuricemia and coronary heart disease: a systematic review and meta-analysis. Arthritis Care Res. 2010;62(2):170-80.

3. Hsu PF, Chuang SY, Yu WC, Hb L, Chan WL, Chen CH. The impacts of serum uric acid on arterial hemodynamics and cardiovascular risks. Acta Cardiol Sin. 2013;29(2):142-50.

4. Letsas KP, Korantzopoulos P, Filippatos GS, Mihas CC, Markou V, Gavrielatos $\mathrm{G}$, et al. Uric acid elevation in atrial fibrillation. Hellenic J Cardiol. 2010;51(3):209-13.

5. Niskanen LK, Laaksonen DE, Nyyssönen K, Alfthan G, Lakka HM, Lakka TA, et al. Uric acid level as a risk factor for cardiovascular and all-cause mortality in middle-aged men: a prospective cohort study. Arch Intern Med. 2004;164(14):1546-51.

6. Kuo CF, See LC, Luo SF, Ko YS, Lin YS, Hwang JS, et al. Gout: an independent risk factor for all-cause and cardiovascular mortality. Rheumatology. 2009;49(7):1422.

7. Karagiannis A, Mikhailidis DP, Tziomalos K, Sileli M, Savvatianos S, Kakafika A, et al. Serum uric acid as an independent predictor of early death after acute stroke. Circ J. 2007;71(7):1120-7.

8. Filippatos GS, Ahmed MI, Gladden JD, Mujib M, Aban IB, Love TE, et al. Hyperuricaemia, chronic kidney disease, and outcomes in heart failure: potential mechanistic insights from epidemiological data. Eur Heart J. 2011;32(6):712-20.

9. Akgul O, Uyarel $H_{1}$, Pusuroglu H, Gul M, Isiksacan N, Turen $\mathrm{S}$, et al. Predictive value of elevated uric acid in turkish patients undergoing primary angioplasty for ST elevation myocardial infarction. Acta Cardiol Sin. 2014;30(2):119-27.

10. Kanbay M, Yilmaz MI, Sonmez A, Solak Y, Saglam M, Cakir E, et al. Serum uric acid independently predicts cardiovascular events in advanced nephropathy. Am J Nephrol. 2012;36(4):324-31.

11. Mitsuhashi $H$, Yatsuya $H$, Matsushita $K$, Zhang H, Otsuka R, Muramatsu T, et al. Uric acid and left ventricular hypertrophy in Japanese men. Circ J. 2009;73(4):667-72.

12. Cicoira M, Zanolla L, Rossi A, Golia G, Franceschini L, Brighetti G, et al. Elevated serum uric acid levels are associated with diastolic dysfunction in patients with dilated cardiomyopathy. Am Heart J. 2002;143(6):1107-11.

13. Abbott RD, Brand FN, Kannel WB, Castelli WP. Gout and coronary heart disease: the Framingham Study. J Clin Epidemiol. 1988;41(3):237-42.
14. Chen SY, Chen CL, Shen ML. Severity of gouty arthritis is associated with Q-wave myocardial infarction: a large-scale, cross-sectional study. Clin Rheumatol. 2007;26(3):308-13.

15. Full LE, Ruisanchez C, Monaco C. The inextricable link between atherosclerosis and prototypical inflammatory diseases rheumatoid arthritis and systemic lupus erythematosus. Arthritis Res Ther. 2009;11(2):217.

16. Wallace SL, Robinson H, Masi AT, Decker JL, Mccarty DJ. Preliminary criteria for the classification of the acute arthritis of primary gout. Arthritis Rheum. 1977;20(3):895-900.

17. Lang RM, Bierig M, Devereux RB, Flachskampf FA, Foster E, Pellikka PA, et al. Recommendations for chamber quantification: a report from the American Society of Echocardiography's Guidelines and Standards Committee and the Chamber Quantification Writing Group, developed in conjunction with the European Association of Echocardiography, a branch of the European Society of Cardiology. J Am Soc Echocardiogr. 2005;18(12):1440-63.

18. Nagueh SF, Appleton CP, Gillebert TC, Marino PN, Oh JK, Smiseth OA, et al. Recommendations for the evaluation of left ventricular diastolic function by echocardiography. Eur J Echocardiogr. 2009;10(2):165-93.

19. Triposkiadis F, Tentolouris K, Androulakis A, Trikas A, Toutouzas K, Kyriakidis $M$, et al. Left atrial mechanical function in the healthy elderly: new insights from a combined assessment of changes in atrial volume and transmitral flow velocity. J Am Soc Echocardiogr. 1995;8(6):801-9.

20. Abhayaratna WP, Seward JB, Appleton CP, Douglas PS, Oh JK, Tajik AJ, et al. Left atrial size: physiologic determinants and clinical applications. J Am Coll Cardiol. 2006;47(12):2357-63.

21. Leung DY, Boyd A, Ng AA, Chi C, Thomas L. Echocardiographic evaluation of left atrial size and function: current understanding, pathophysiologic correlates, and prognostic implications. Am Heart J. 2008;156(6):1056-64.

22. Khouri SJ, Maly GT, Suh DD, Walsh TE. A practical approach to the echocardiographic evaluation of diastolic function. J Am Soc Echocardiogr. 2004;17(3):290-7.

23. Aurigemma GP, Gaasch WH. Diastolic heart failure. N Engl J Med. 2004:351(11):1097-105.

24. Douglas PS. The left atrium: a biomarker of chronic diastolic dysfunction and cardiovascular disease risk. J Am Coll Cardiol. 2003;42(7):1206-7.

25. Pritchett AM, Mahoney DW, Jacobsen SJ, Rodeheffer RJ, Karon BL, Redfield MM. Diastolic dysfunction and left atrial volume: a population-based study J Am Coll Cardiol. 2005;45(1):87-92.

26. Sherazi S, Zaręba W. Diastolic heart failure: predictors of mortality. Cardiol J. 2011;18(3):222-32.

27. Tsang TS, Gersh BJ, Appleton CP, Tajik AJ, Barnes ME, Bailey KR, et al. Left ventricular diastolic dysfunction as a predictor of the first diagnosed nonvalvular atrial fibrillation in 840 elderly men and women. J Am Coll Cardiol. 2002;40(9):1636-44.

28. From AM, Scott CG, Chen HH. The development of heart failure in patients with diabetes mellitus and pre-clinical diastolic dysfunction: a populationbased study. J Am Coll Cardiol. 2010;55:300-5.

29. Krishnan E, Hariri A, Dabbous O, Pandya BJ. Hyperuricemia and the echocardiographic measures of myocardial dysfunction. Congest Heart Fail. 2012;18(3):138-43.

30. Torre-Amione G. Immune activation in chronic heart failure. Am J Cardiol. 2005;95(11):3-8

31. Mann DL. Inflammatory mediators and the failing heart past, present, and the foreseeable future. Circ Res. 2002;91(11):988-98.

32. Rivas-Gotz C, Manolios M, Thohan V, Nagueh SF. Impact of left ventricular ejection fraction on estimation of left ventricular filling pressures using tissue Doppler and flow propagation velocity. Am J Cardiol. 2003;91(6):780-4.

33. Ommen SR, Nishimura RA, Appleton CP, Miller FA, Oh JK, Redfield MM, et al. Clinical utility of Doppler echocardiography and tissue Doppler imaging in the estimation of left ventricular filling pressures a comparative simultaneous Doppler-catheterization study. Circulation. 2000;102(15):1788-94.

34. Szardien S, Nef HM, Voss S, Troidl C, Liebetrau C, Hoffmann J, et al. Regression of cardiac hypertrophy by granulocyte colony-stimulating factorstimulated interleukin-1 $\beta$ synthesis. Eur Heart J. 2011;33(5):595-605.

35. Appleton CP, Galloway JM, Gonzalez MS, Gaballa M, Basnight MA. Estimation of left ventricular filling pressures using two-dimensional and Doppler echocardiography in adult patients with cardiac disease. Additional value of analyzing left atrial size, left atrial ejection fraction and the difference in duration of pulmonary venous and mitral flow velocity at atrial contraction. J Am Coll Cardiol. 1993;22(7):1972-82. 